\title{
RELATIVE PERIODIC ORBITS IN TRANSITIONAL PIPE FLOW
}

\author{
Yohann Duguet*, Chris C.T. Pringle, and Rich R. Kerswell \\ School of Mathematics, University of Bristol \\ BS8 1TW, Bristol, United Kingdom
}

\begin{abstract}
A dynamical system description of the transition process in shear flows with no linear instability starts with a knowledge of exact coherent solutions, among them travelling waves (TWs) and relative periodic orbits (RPOs). We describe a numerical method to find such solutions in pipe flow and apply it in the vicinity of a Hopf bifurcation from a TW which looks to be especially relevant for transition. The dominant structural feature of the RPO solution is the presence of weakly modulated streaks. This RPO, like the TW from which it bifurcates, sits on the laminar-turbulent boundary separating initial conditions which lead to turbulence from those which immediately relaminarise.
\end{abstract}

\footnotetext{
* Present address : Linné Flow Centre, KTH Mechanics, SE-100 44 Stockholm, Sweden.
} 


\section{INTRODUCTION}

Ever since the experiments of Osborne Reynolds in $1883, \frac{1}{2}$ it has been known that the flow of a Newtonian fluid in a circular pipe undergoes an abrupt transition from a laminar to a turbulent state. This transition is governed by one dimensionless parameter, the Reynolds number $R e:=U d / \nu$ ( $U$ is the bulk velocity, $d$ the pipe diameter and $\nu$ the kinematic viscosity of the fluid). Transition is usually observed experimentally for $R e \sim 2000 \pm 250$ but can be delayed to larger values in especially well-controlled experiments. $\stackrel{2}{2} \underset{2}{*}$ The laminar state (Hagen-Poiseuille flow) is believed linearly stable for all Re, so that transition to turbulence cannot be explained by an ordered sequence of bifurcations starting from the basic state. Instead, pipe flow belongs to a more general class of parallel shear flows, including plane Couette and plane Poiseuille flow, which undergo finite-amplitude instability. $\underline{\underline{4}}$ The transitional dynamics of these flows then appears organised around the presence of other

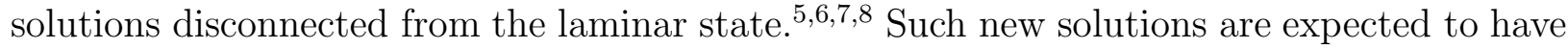
a relatively simple structure compared to the turbulent dynamics and to be unstable. These expectations are conceptually satisfying for two reasons. Firstly, relatively simple threedimensional mechanisms (e.g. the 'self-sustaining process') have been identified in low-Re flows ${ }^{9,10}$ allowing turbulence to be sustained over long times, which evokes the possibility of organised dynamics. Secondly, instability is needed to rationalise why neither steady nor periodic exact states are ever found as limiting states to emerge from an initially turbulent flow. The fact that statistically recurrent states have been clearly observed, e.g. streaky velocity fields with an intrinsic wavelength in most wall-bounded shear flows, $\frac{9}{,}$ is most easily explained by imagining repeated visits in phase space to the vicinity of exact solutions of the governing equations.

Progress in numerical computation has first led to the discovery of some exact solutions in plane shear flows $5,10,11$ and more recently pipe flow. $\stackrel{12,13}{ }$ They are all equilibria (steady states) or relative equilibria (travelling waves which are steady in a moving frame) and all possess a small number of unstable eigendirections. They appear through saddle-node bifurcations and are disconnected from the laminar state. There is a tremendous interest in these solutions as very similar structures have been observed transiently in experiments. $\stackrel{14,15}{1}$ It is worth emphasizing that their simple dynamics (steadiness or constant speed propagation) is inherent to the method used to seek them, and undoubtedly hides a larger variety of other 
solutions. At some point the physical description of the flow in terms of three-dimensional structures ceases to be enlightening and it is useful to think in terms of a trajectory in phase space. Formally, the Navier-Stokes equations can be projected onto any infinite-dimensional basis of incompressible velocity fields to yield an autonomous dynamical system of the form $d \boldsymbol{X} / d t=\boldsymbol{F}(\boldsymbol{X}, R e)$. The choice of the basis, and hence the particular projection, is not crucial. The topology of the phase space associated to pipe flow near transition is thought to be organised around one locally attractive point (the 'laminar' flow) and a chaotic saddle, i.e. a set of unstable solutions and their heteroclinic and homoclinic connections $. \underline{6}, \underline{12}$ From this point of view, it is necessary to know which states the saddle is made of in order to understand the dynamics near transition. It has been demonstrated that the unstable solutions known so far in pipe flow, the travelling waves (TWs), are visited only for about $O(10-20 \%)$ of the time. $\stackrel{16}{17}$ This indicates that, provided the picture is correct, more solutions of a different type have to be sought. The next level up in the hierarchy of solutions from relative equilibria is relative periodic orbits (RPOs). They are an extension of periodic orbits (in the same way as traveling waves correspond to degenerate fixed points) due to the invariance of the equations with respect to the azimuthal and axial directions. Physically, a RPO corresponds to a flow which repeats itself periodically in a given moving frame, translating and/or rotating along the pipe axis at a constant rate. In the cylindrical coordinate system $(s, \theta, z)$ aligned with the pipe axis, it is a time-dependent velocity field $\boldsymbol{v}(s, \theta, z, t)$ satisfying

$$
\boldsymbol{v}(s, \theta, z, t+T)=\boldsymbol{v}(s, \theta+\Delta \theta, z+\Delta z, t)
$$

for some constants $T, \Delta \theta$ and $\Delta z$. The initial motivation behind the search for periodic behaviour is Periodic Orbit Theory $\underset{18}{18}$ which states that any dynamical average of a smooth chaotic dynamical system can be evaluated in a deterministic way by finding all unstable periodic orbits of the system up to a certain period, and carefully averaging over them. Consideration of continuous symmetries in the original PDEs - here translation in $z$ and rotation in $\theta$ - extends this expansion over all relative periodic orbits. 19 Even if complete knowledge of all periodic orbits of the system seems computationally ambitious today, a start needs to be made in developing numerical tools to find them in anticipation of greater computing power tomorrow. Some progress has already been made in plane Couette flow with the recent discovery of periodic ${ }^{20}$ and relative periodic orbits ${ }^{21}$ embedded in turbulence, 
albeit in small flow domains. Beyond the obvious computational savings, restricting the largest wavelength in the flow has proved a very useful tool for isolating key dynamics ${ }^{9}$. In the same spirit, we will here concentrate on relatively short 'periodic' pipes only a few diameters long.

In this study we are interested in RPOs which are located in the laminar-turbulent boundary, the invariant subset of phase-space on which trajectories neither relaminarise nor become turbulent. States belonging to this subset can be visited transiently by phase-space trajectories during the transition process. Phase-space trajectories constrained to remain precisely on this laminar-turbulent boundary have been found to approach a chaotic attractor ${ }^{38}$ centered on one particular TW solution, the 'asymmetric TW' found by Pringle \& Kerswell. $\stackrel{22}{2}$ The importance of this particular TW has been confirmed by further calculations which show that trajectories constrained to lie on this laminar-turbulent boundary recurrently approach this TW $\underline{\underline{23}}$ We therefore concentrate on looking for RPOs which bifurcate from this asymmetric TW.

The paper is organised as follows. Section II explains in detail the numerical method chosen to find RPOs from a starting guess and section III describes the new branch of RPOs found. Subsection IV confirms that the RPO is on the laminar-turbulent boundary and then explores the likelihood of connections between the RPO and other TWs. Section V discusses the relevance of the RPO to the transition process as well as the numerical limitations of the method.

\section{NUMERICAL PROCEDURE}

\section{A. Governing equations}

We consider the incompressible flow of Newtonian fluid in a cylindrical pipe and adopt the usual set of cylindrical coordinates $(s, \theta, z)$ and velocity components $\boldsymbol{u}=u \hat{\boldsymbol{s}}+v \hat{\boldsymbol{\theta}}+w \hat{\boldsymbol{z}}$. Units of length and flow speed are taken as the pipe radius and the bulk speed $U$. The computational domain is $(s, \theta, z) \in[0,1] \times[0,2 \pi] \times[0, L]$, where $L=2 \pi / \alpha$ is the length of the pipe. The non-dimensionalised incompressible three-dimensional Navier-Stokes equations 
read

$$
\begin{gathered}
\frac{\partial \boldsymbol{u}}{\partial t}+(\boldsymbol{u} \cdot \boldsymbol{\nabla}) \boldsymbol{u}=-\nabla p+\frac{1}{R e} \nabla^{2} \boldsymbol{u}, \\
\boldsymbol{\nabla} \cdot \boldsymbol{u}=0
\end{gathered}
$$

where $R e:=U d / \nu$. The flow is driven by a constant mass-flux condition

$$
1 / \pi \int_{0}^{1} \int_{0}^{2 \pi} w s d s d \theta=1
$$

as in recent experiments. $\underline{2}, \underline{24}, 25$ Although time is calculated in units of $d / 2 U$, all times are quoted hereafter in the usual units of $d / U$. The boundary conditions are periodicity across the pipe length $\boldsymbol{u}(s, \theta, z)=\boldsymbol{u}(s, \theta, z+L)$ and no-slip on the walls $\boldsymbol{u}(1, \theta, z)=\mathbf{0}$. In the non-dimensionalisation used, the expression of the Hagen-Poiseuille flow is $\boldsymbol{u}_{H P}=2\left(1-s^{2}\right) \hat{\boldsymbol{z}}$.

\section{B. Time-stepping code}

The basic tool for the numerical determination of periodic orbits is an accurate timestepping code. The direct numerical simulation code written by A.P. Willis ${ }^{26,27,28}$ was adopted which is based upon the toroidal-poloidal representation of the velocity field 29

$$
\boldsymbol{u}=\nabla \times(\Psi \hat{\boldsymbol{z}})+\nabla \times \nabla \times(\Phi \hat{\boldsymbol{z}})
$$

The two scalar potentials $\Phi$ and $\Psi$ are discretised using high-order finite differences in the radial direction $s$, and with spectral Fourier expansions in the azimuthal $\theta$ and axial $z$ directions. At any discrete radial location $s_{j},(j=1, \ldots, N)$, each of the potentials (e.g. here $\Phi)$ is therefore discretised according to the formula:

$$
\Phi\left(s_{j}, \theta, z, t ; \alpha\right)=\sum_{k=-K}^{K} \sum_{m=-M}^{M} \Phi_{j k m}(t) e^{i(m \theta+\alpha k z)}
$$

The resolution of a given calculation is described by a triplet $(N, M, K)$. The phase-space associated to the Navier-Stokes equations is the set of complex coefficients $\left\{\Phi_{j k m}, \Psi_{j k m}\right\}$, which corresponds to a dynamical system with $n \sim 8 M N K$ real degrees of freedom (typically $\left.n=O\left(10^{5}\right)\right)$. Its metric is defined by the Euclidean scalar product $\langle$,$\rangle . A shift back in$ physical space by $(\Delta z, \Delta \theta)$ corresponds in phase-space to the transformation:

$$
\left(\Psi_{j k m}, \Phi_{j k m}\right) \rightarrow\left(\Psi_{j k m}, \Phi_{j k m}\right) e^{-i(m \Delta \theta+\alpha k \Delta z)}
$$


Time discretisation is of second-order, using a Crank-Nicholson scheme for the diffusion term and an Euler predictor step for the non-linear terms. In this study we confine our calculations to a pipe of length $L=2 \pi / 0.75 \sim 8.38$ radii $=4.19 d$. This length has been chosen because it is a wavelength for which the asymmetric $\mathrm{TW}^{22}$ is known to be on the laminar-turbulent boundary and for which most data were already available.

\section{The Newton-Krylov method}

\section{Periodic orbits}

A periodic orbit (of period $T$ ) of a dynamical system $\dot{\boldsymbol{X}}=\boldsymbol{F}(\boldsymbol{X})$ is sought as a solution $(\boldsymbol{X}, T)$ of the equation $\boldsymbol{G}(\boldsymbol{X})=\mathbf{0}$, where

$$
\boldsymbol{G}(\boldsymbol{X}):=\phi^{T}(\boldsymbol{X})-\boldsymbol{X}
$$

Here $\phi^{t}(\boldsymbol{X})$ refers to the point at time $t>0$ on the trajectory starting from $\boldsymbol{X}$ at time $t=0$. A value of the period $t=T$ has to be chosen to uniquely define $\boldsymbol{G}(\boldsymbol{X})$. We choose $T$ as the time minimising the $\boldsymbol{X}$-dependent scalar function $g: t \rightarrow\left|\phi^{t}(\boldsymbol{X})-\boldsymbol{X}\right|^{2}$ over a given time interval. This is done by accurate interpolation of the zeros of its derivative, the scalar function $g^{\prime}: t \rightarrow 2\left\langle\phi^{t}(\boldsymbol{X})-\boldsymbol{X}, \partial \phi^{t}(\boldsymbol{X}) / \partial t\right\rangle$. Figure 1 shows schematically that the time $t=T$ is picked up along the trajectory when its tangent vector is orthogonal to the difference vector $\phi^{t}(\boldsymbol{X})-\boldsymbol{X}$, which ensures that the trajectory has come back closest to the initial point. A standard method to search for zeros of $\boldsymbol{G}$ in low-dimensional systems (typically $n \lesssim O\left(10^{3}\right)$ ) is the Newton-Raphson method. This iterative algorithm, based on successive Taylor expansions of $\boldsymbol{G}$, produces a sequence of iterates $\boldsymbol{X}^{(k)}, k \geq 0$ defined by

$$
\boldsymbol{X}^{(k+1)}=\boldsymbol{X}^{(k)}+\boldsymbol{\delta} \boldsymbol{X}^{(k)},
$$

where

$$
\boldsymbol{J}\left(\boldsymbol{X}^{(k)}\right) \boldsymbol{\delta} \boldsymbol{X}^{(k)}=-\boldsymbol{G}\left(\boldsymbol{X}^{(k)}\right),
$$

$\boldsymbol{J}$ being the Jacobian matrix associated to $\boldsymbol{G}$. When no analytical expression for $\boldsymbol{J}$ is available, it is generally computed by finite differences, at the cost of one evaluation of $\boldsymbol{G}$ 


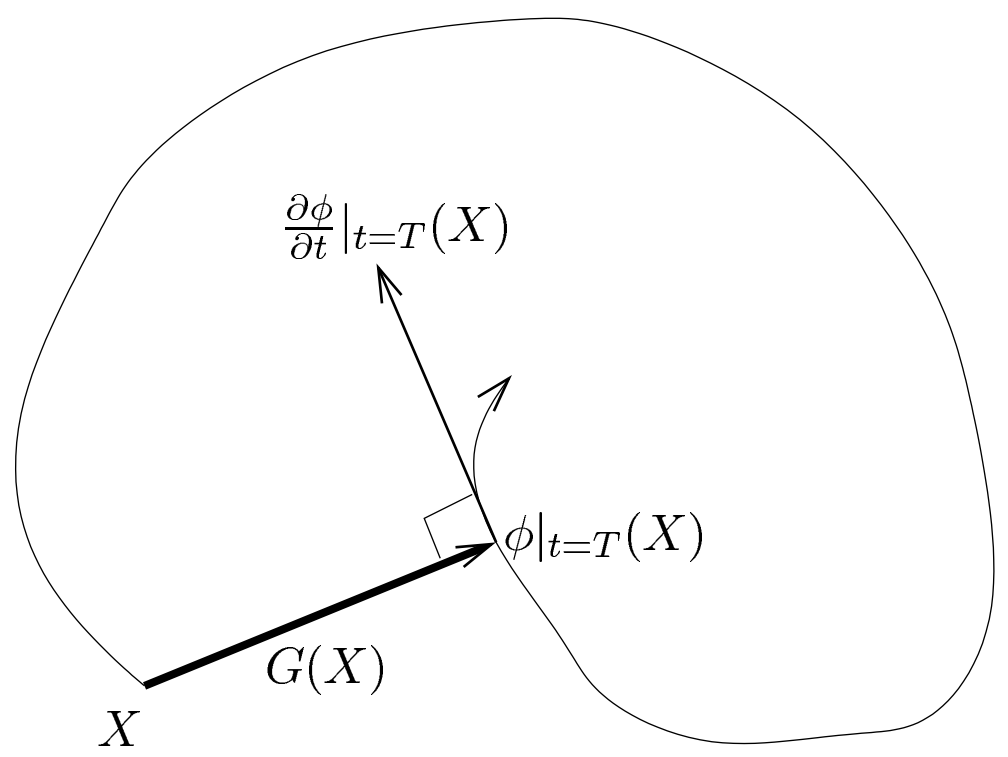

FIG. 1: Schematic view of phase-space. Definition of the functional $\boldsymbol{G}$ to minimise in order to find exact periodic orbits.

for each column of the matrix. In very high dimension $n \gtrsim O\left(10^{4}\right)$, however, the time needed for $n+1$ evaluations of $\boldsymbol{G}$, as well as the storage of $\boldsymbol{J}$ (not to mention solving equation (100), can become prohibitive, thus we turn our attention towards matrix-free Inexact Newton-Krylov methods.

At every Newton step $k$, we solve the system (10) using the GMRES algorithm. $\frac{30}{\text { This }}$ iterative linear solver only requires matrix-vector products that can be evaluated using finite differences, since for a vector $\boldsymbol{p}$ and $\epsilon$ sufficiently small (e.g. $10^{-7}$ ) we have:

$$
\boldsymbol{J} \boldsymbol{p}=\frac{\boldsymbol{G}\left(\boldsymbol{X}^{(k)}+\epsilon \boldsymbol{p}\right)-\boldsymbol{G}\left(\boldsymbol{X}^{(k)}\right)}{\epsilon}+O(\epsilon) .
$$

Hence no matrix needs to be stored explicitly. Using (11), GMRES iteratively builds an orthogonal basis of the Krylov subspace spanned by $\left\{\boldsymbol{r}_{0}, \boldsymbol{J} \boldsymbol{r}_{0}, \boldsymbol{J}^{2} \boldsymbol{r}_{0}, \ldots\right\}$, starting from $\boldsymbol{r}_{0}=$ $-\boldsymbol{G}\left(\boldsymbol{X}^{(k)}\right)$. At each GMRES step, a Gram-Schmidt procedure reorthonormalises the Krylov subspace producing a basis $\left\{\boldsymbol{V}_{0}, \boldsymbol{V}_{1}, \boldsymbol{V}_{2}, \ldots\right\}$. Then an approximation to the solution of (10) is constructed on this basis, until convergence is decided according to the criterion:

$$
\left|\boldsymbol{J}^{(k)} \boldsymbol{\delta} \boldsymbol{X}^{(k)}+\boldsymbol{G}^{(k)}\right| \leq \eta^{(k)}\left|\boldsymbol{G}^{(k)}\right|
$$

The 'forcing' constant $\eta^{(k)}$ appearing in formula (12) is ideally zero, and numerically it should be in principle very small. However, non-vanishing values of $\eta$ can allow for a much 
quicker convergence of the overall Newton scheme, by avoiding useless oversolving. Values of $\eta \sim O\left(10^{-1}\right)$ have produced faster convergence and even better performances were observed when updating $\eta^{(k)}$ with the choice 2 of Eisenstat \& Walker. $\underline{\underline{31}}$

\section{Double dogleg step}

Newton-Raphson algorithms are known to converge only if the initial guess is sufficiently close to a zero of the function $\boldsymbol{G}$, which in high dimension often means no convergence at all. Moreover, even close to a solution, Newton-Raphson steps either can be too large or nearly orthogonal to the gradient of $|\boldsymbol{G}|^{2}$, in which case the algorithm stagnates and the classical strategy of linear backtracking (also called 'damped Newton') is of no help $\underline{\underline{32}}$ To remedy this, it is useful to embed the algorithm into what is commonly called a 'globally' convergent strategy. Here 'global' does not mean that convergence is achieved whatever the starting point, but instead that the basin of attraction of a solution is reasonably enlarged. We adopt the 'double dogleg step' method proposed by Dennis and Schnabel, $\underline{\underline{33}}$ which is a subclass of the 'trust region' algorithms (see Viswanath $\underline{21}$ for an implementation of another subclass call the 'hook step' approach). All these methods start from the knowledge of the Newton step $\boldsymbol{\delta} \boldsymbol{X}_{N}$ (here we omit the superscript $(k)$ ), and aim at constructing a new vector $\boldsymbol{\delta} \boldsymbol{X}$ whose norm is bounded by a 'trust length' $\delta_{c}$. The way $\boldsymbol{\delta} \boldsymbol{X}$ is chosen is described in the next paragraph. At the first stage of the loop, $\boldsymbol{\delta} \boldsymbol{X}=\boldsymbol{\delta} \boldsymbol{X}_{N}$ and $\delta_{c}=\left|\boldsymbol{\delta} \boldsymbol{X}_{N}\right|$. Then at every iteration of the trust region algorithm, a check is made to see whether $\left|\boldsymbol{G}\left(\boldsymbol{X}^{(\boldsymbol{k})}+\boldsymbol{\delta} \boldsymbol{X}\right)\right|$ is sufficiently smaller than $\left|\boldsymbol{G}\left(\boldsymbol{X}^{(k)}\right)\right|$. If it is, then the loop is finished and we set $\boldsymbol{X}^{(k+1)}$ to $\boldsymbol{X}^{(k)}+\boldsymbol{\delta} \boldsymbol{X}$; if not, then $\delta_{c}$ is divided by 2 , a new vector $\boldsymbol{\delta} \boldsymbol{X}$ is computed and the process is repeated.

In the case of the double dogleg step, two descent directions for $|\boldsymbol{G}|^{2}$ are used to generate the new vector $\boldsymbol{\delta} \boldsymbol{X}$ : the first one is the Newton step $\boldsymbol{\delta} \boldsymbol{X}_{N}$, the second is the steepest descent direction (see figure 2):

$$
-\frac{1}{2} \nabla|\boldsymbol{G}|^{2}=-\boldsymbol{J}^{t} \boldsymbol{G},
$$

where $\boldsymbol{J}^{t}$ is the transpose of the Jacobian matrix $\boldsymbol{J}$. We can define the Cauchy Point $\boldsymbol{X}^{(k)}+\boldsymbol{\delta} \boldsymbol{X}_{C P}$, where the vector $\boldsymbol{\delta} \boldsymbol{X}_{C P}$ minimizes the quadratic form $\boldsymbol{\delta} \boldsymbol{X} \rightarrow|\boldsymbol{J} \boldsymbol{\delta} \boldsymbol{X}+\boldsymbol{G}|^{2}$ along the steepest descent direction (whereas the overall minimizer of this quadratic form 
defines the Newton step $\boldsymbol{\delta} \boldsymbol{X}_{N}$ ). Projection of $\boldsymbol{\delta} \boldsymbol{X}_{C P}$ onto the Krylov space (already built to find $\boldsymbol{\delta} \boldsymbol{X}_{N}$ at the same Newton iteration) allows a low-cost approximation of $\boldsymbol{\delta} \boldsymbol{X}_{C P}$, as long as the dimension of the Krylov subspace itself is low. If the $\boldsymbol{V}_{i} \mathrm{~s}$ form an orthonormal basis of the Krylov subspace and $K$ is its dimension, we have:

$$
\boldsymbol{\nabla}|\boldsymbol{G}|^{2} \sim \sum_{i=1}^{K}\left\langle\boldsymbol{J}^{t} \boldsymbol{G}, \boldsymbol{V}_{i}\right\rangle \boldsymbol{V}_{i}=\sum_{i=1}^{K}\left\langle\boldsymbol{G}, \boldsymbol{J} \boldsymbol{V}_{i}\right\rangle \boldsymbol{V}_{i}
$$

In (14), the scalar coefficients $\left\langle\boldsymbol{G}, \boldsymbol{J} \boldsymbol{V}_{i}\right\rangle$ are already known up for $1 \leq i \leq K$ since (normalized by $-\left|\boldsymbol{r}_{0}\right|$ ) they form the first row of the Hessenberg matrix used for the GMRES inversion. $\underline{30}$

Finally, given $\boldsymbol{\delta} \boldsymbol{X}_{N}$ and $\boldsymbol{\delta} \boldsymbol{X}_{C P}$, the double dogleg algorithm finds the intersection between the piecewise linear curve linking the Cauchy Point to $\boldsymbol{X}+\mu \boldsymbol{\delta} \boldsymbol{X}_{N}$, and the ball of radius $\delta_{c}$. So the new trial vector is chosen as:

$$
\boldsymbol{\delta} \boldsymbol{X}=\boldsymbol{\delta} \boldsymbol{X}_{C P}+\lambda_{c}\left(\mu \boldsymbol{\delta} \boldsymbol{X}_{N}-\boldsymbol{\delta} \boldsymbol{X}_{C P}\right)
$$

where $\mu$ is a constant set to 0.8 (following ref. ${ }^{33}$ ) and $\lambda_{c}>0$ is chosen such that $|\boldsymbol{\delta} \boldsymbol{X}|=\delta_{c}$. If $\left|\delta_{c}\right| \leq\left|\boldsymbol{\delta} \boldsymbol{X}_{C P}\right|$, the dogleg curve along which optimisation is achieved is simply the steepestdescent direction of $|\boldsymbol{G}|^{2}$.

\section{Relative periodic orbits}

Allowing for shifts in $\theta$ and $z$ implies a modification of the function $\boldsymbol{G}$. If we seek a relative periodic orbit satisfying $\boldsymbol{v}(r, \theta, z, t+T)=\boldsymbol{v}(r, \theta+\Delta \theta, z+\Delta z, t)$ for any $t$, we have to change $\boldsymbol{G}(\boldsymbol{X})$ into $\boldsymbol{G}(\boldsymbol{X})=\phi_{-\Delta \theta,-\Delta z}^{T}(\boldsymbol{X})-\boldsymbol{X}$. In this expression, $\phi^{T}(\boldsymbol{X})$ is shifted back in the $\theta$ - and $z$-directions using (7), before being compared to the initial point of the

orbit. $T$ is chosen as the time minimising the new $|\boldsymbol{G}|^{2}$, whereas $\Delta \theta$ and $\Delta z$ are considered as two extra variables of the Newton algorithm: ${ }^{21}$ we define a $(n+2)$-dimensional vector $\boldsymbol{X}^{+}=(\boldsymbol{X}, \Delta \theta, \Delta z)$ and the function $\boldsymbol{G}^{+}: R^{n+2} \rightarrow R^{n+2}$ such that

$$
\boldsymbol{G}_{i}^{+}\left(\boldsymbol{X}^{+}\right)=\boldsymbol{G}_{i}(\boldsymbol{X}),(i=1, . ., n),
$$

while the two last components of $\boldsymbol{G}^{+}$are defined by the scalar products in $R^{n}$ :

$$
\boldsymbol{G}_{n+1}^{+}\left(\boldsymbol{X}^{+}\right)=\left\langle\boldsymbol{G}, \frac{\partial \phi^{T}}{\partial \Delta \theta}\right\rangle
$$




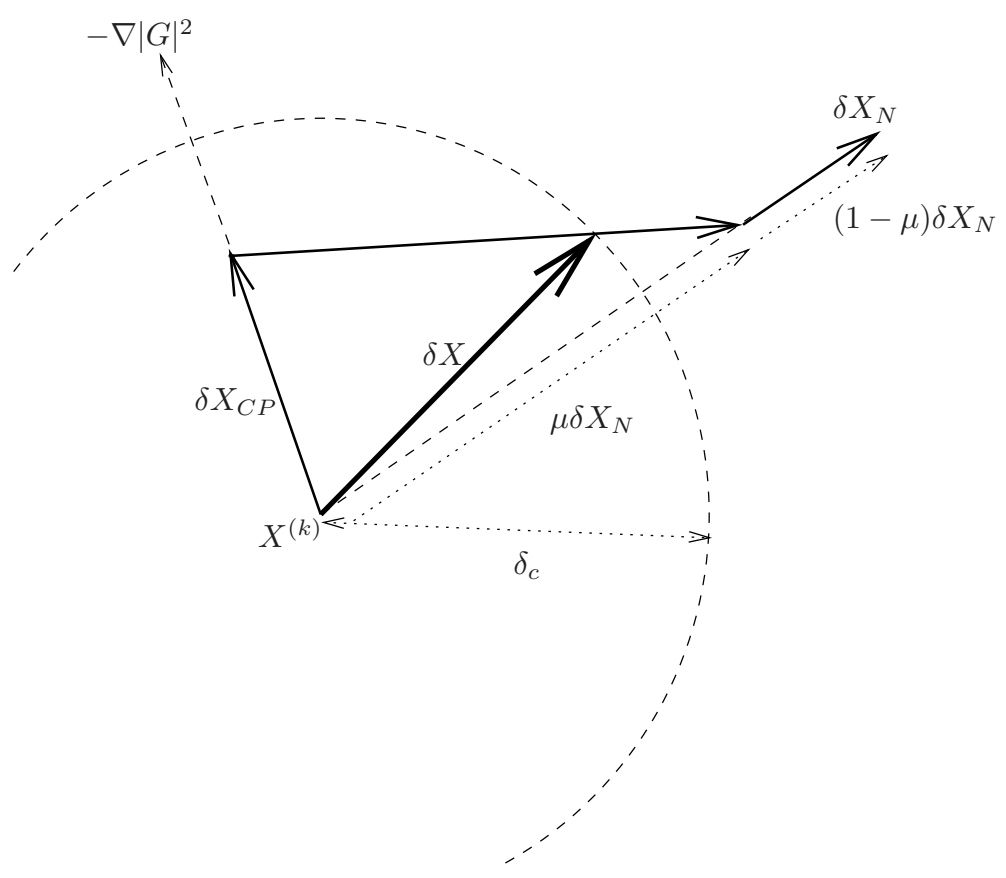

FIG. 2: Schematic view of the double dogleg technique (adapted from Dennis \& Schnabel ${ }^{33}$ ) in phase space. $\boldsymbol{X}^{(k)}$ is the state at the $k^{t h}$ Newton iteration, $\boldsymbol{\delta} \boldsymbol{X}_{N}$ represents the Newton step computed using the inexact GMRES method, and $-\boldsymbol{\nabla}|\boldsymbol{G}|^{2}$ is the steepest descent direction of the residual from $\boldsymbol{X}^{(k)}$. At a given step, a new state $\boldsymbol{X}^{(k+1)}=\boldsymbol{X}^{(\boldsymbol{k})}+\boldsymbol{\delta} \boldsymbol{X}$ is suggested as the intersection between the double dogleg curve (medium thick line) and the ball of radius $\delta_{c}$.

$$
\boldsymbol{G}_{n+2}^{+}\left(\boldsymbol{X}^{+}\right)=\left\langle\boldsymbol{G}, \frac{\partial \phi^{T}}{\partial \Delta z}\right\rangle \text {. }
$$

These two derivatives are easily computed by central finite differences. Starting from a guess $\boldsymbol{X}_{0}$ (which includes a starting point in phase space as well as two initial values of $\Delta \theta$ and $\Delta z)$, the Newton-Krylov method is now applied to locate zeros of the function $\boldsymbol{G}^{+}$. It is worth reiterating that although $T$ looks to be on the same footing as $\Delta \theta$ and $\Delta z$, this is not the case. The condition $<\boldsymbol{G}, \partial \phi^{t} / \partial t>=0$ which defines $T$ is imposed at every Newton/dogleg iteration whereas the equivalent conditions for $\Delta \theta$ and $\Delta z$ are only truly reached when convergence to a RPO has been achieved.

Non-rotating TW solutions are a special case of RPOs, when $T$ and $\Delta z$ are linked by the relation $\Delta z=c T$, with $c$ the axial propagation speed of the wave. Because of this degeneracy, TW solutions with zero azimuthal speed can be sought, using the same algorithm, by fixing the value of $\Delta z$ to $2 \pi / \alpha$, and setting $\boldsymbol{G}_{n+2}^{+}=0$. 


\section{RESULTS}

\section{A. Hopf bifurcation of traveling waves}

The Newton-Krylov algorithm described in Section [I was used to find a RPO bifurcating off the asymmetric TW branch in a periodic pipe of length $L \sim 4.19 d(\alpha=0.75)$ where it is known to be embedded in the laminar-turbulent boundary. The asymmetric TW branch originates from a supercritical bifurcation at Re 1770 off a "mirror-symmetric" branch of TWs. ${ }^{22}$ The asymmetric TW propagates axially with a phase speed $c=1.34 U$ but does not rotate. For $R e \sim 1785$, it displays a pair of high-speed streaks sandwiching an eccentric low-speed streak (see Figure 3). A streamwise vortex tube is present in the vicinity of the low-speed streak, ensuring the regeneration of the streak via a lift-up effect. The two highspeed regions extend to the other side of the cross-section, and another low-speed streak of lesser intensity is present on the right of the original one. As Re increases, the solution becomes more asymmetric and the streaks on the right of the Figure tend to disappear.

The numerical resolution chosen in this work is $(N, M, K)=(86,16,5)$ to match that used by Pringle $\&$ Kerswell in the $\theta$ - and $z$-directions. This is sufficient to observe a drop-off of 6 decades in the axial spectrum and more than 8 decades in the radial and azimuthal energy spectra. The rapid drop-off in the axial wavenumbers is typical of lower-branch solutions and their weakly wiggling streak structure. It is even more pronounced as $R e \rightarrow \infty . \underline{34}$ The Navier-Stokes equations have been linearised around the TW solution expressed in the Galilean frame moving with speed $c$ along the axis. The corresponding eigenvalue problem was solved numerically using an Arnoldi routine for $R e$ up to $5000 \stackrel{35}{ }^{\text {There are }}$ 4 unstable eigenvalues ( 2 real and 2 complex conjugate ones) for $R e<R e_{H}=1785.6$ and only 2 real ones for $R e>R e_{H}$ indicating a Hopf bifurcation at $R e=1785.6$ (see Figure 4). The center manifold theorem states that for $R e$ close enough to $R e_{H}$, a periodic orbit must exist in the same moving frame. Its amplitude scales locally like $O\left(\sqrt{\left|R e_{H}-R e\right|}\right)$. The period of the orbit is given by the imaginary part of the Hopf eigenvalue pair, here $T=2 \pi / \operatorname{Im}\left(\lambda_{H}\right) \sim 43 d / U$. A good estimation for the shifts is the distance travelled by the TW in the axial and azimuthal directions during this period, hence $\Delta z^{(0)}=c T \sim 58.7 d$ and $\Delta \theta^{(0)}=0$ (since this wave has no azimuthal phase speed). The starting point used for the Newton-Krylov algorithm is a slight perturbation of the TW along one of the two conjugate 

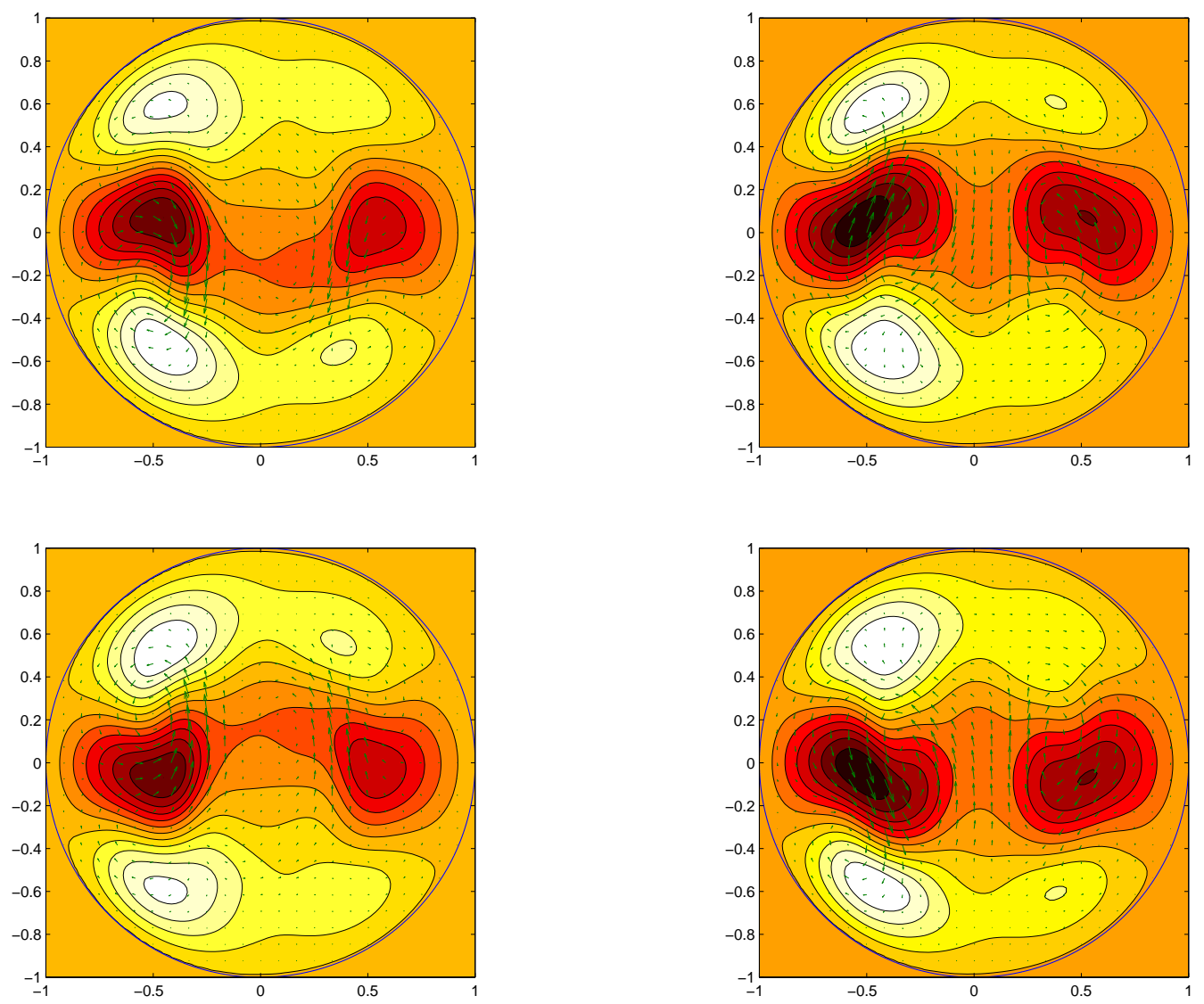

FIG. 3: Velocity profile of the asymmetric TW solution at the Hopf bifurcation $(\alpha=0.75, R e=$ 1785). From left to right, from top to bottom : $z / L=0,0.25,0.5,0.75$. The arrows indicate the cross-stream velocity, and the shading indicates the difference between the streamwise velocity and the laminar profile (light/white indicating positive values and dark/red negative values).

neutral directions, denoted as the eigenvector $\boldsymbol{e}_{H}$ :

$$
\left(\boldsymbol{X}^{+}\right)^{(0)}=\left(\boldsymbol{X}_{T W}+\epsilon \boldsymbol{e}_{H}, \Delta z^{(0)}, \Delta \theta^{(0)}\right)
$$

For values of $\epsilon \sim O\left(10^{-2}\right)$ and $R e_{H}-1<R e<R e_{H}$, the algorithm converged to a RPO, within a residual $r_{\infty}=\left|\boldsymbol{X}_{-\Delta z}(T)-\boldsymbol{X}(0)\right| /|\boldsymbol{X}(0)|$ of $O\left(10^{-6}\right)$. The converged values of the period $T$ and the shift $\Delta z$ are very close to the expected values, while the RPO appears not to rotate $(\Delta \theta=0)$. The new solution branch bifurcates towards the regime where the original branch has fewer stable directions, namely $R e<R e_{H}$. This Hopf bifurcation is hence supercritical, although the new solution branch extends to lower values of $R e$. From now on we will also use the reduced positive parameter $\delta=R e_{H}-R e$. 


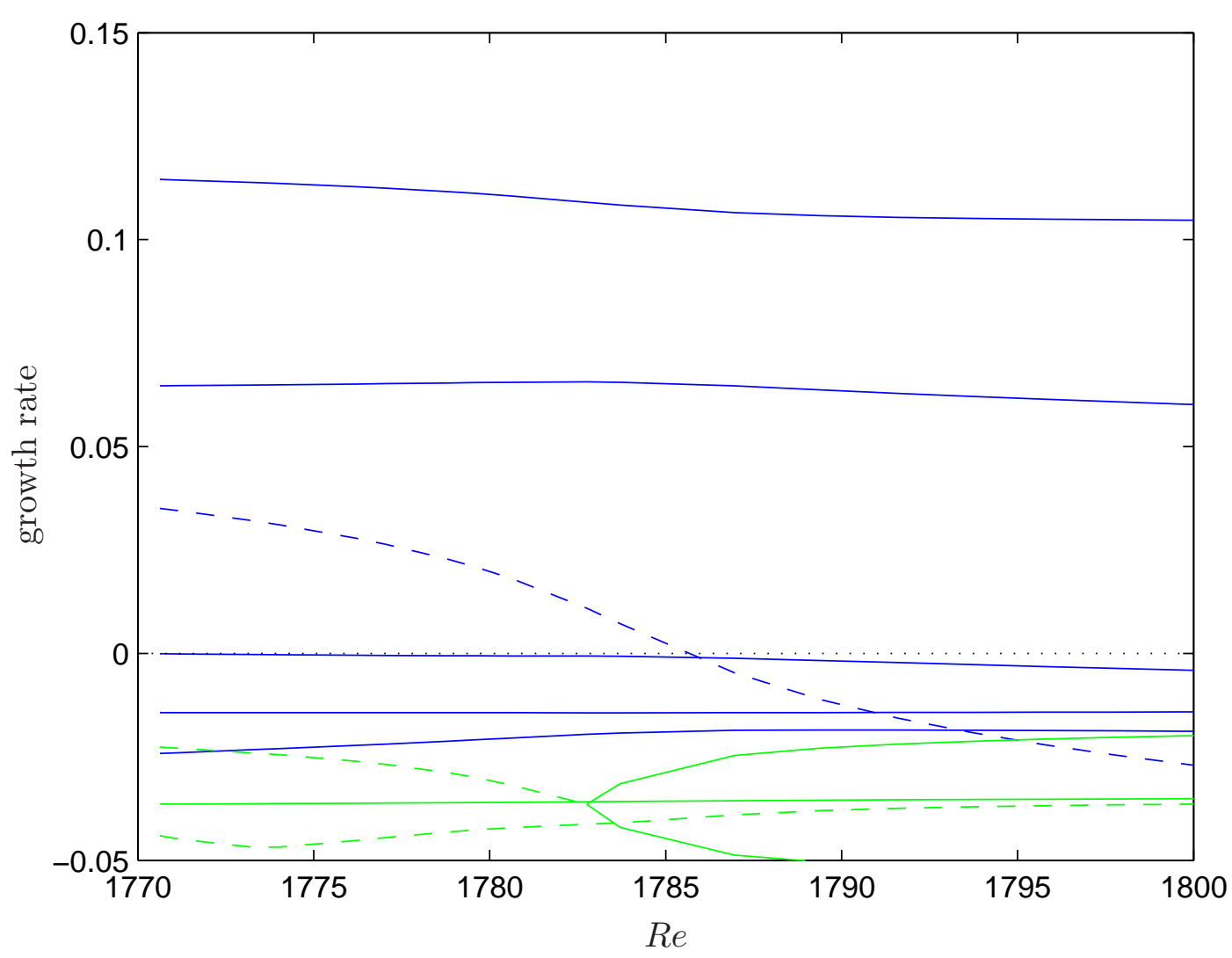

FIG. 4: Stability analysis of the asymmetric TW for $\alpha=0.75$ near the Hopf bifurcation point at $R e=R e_{H}=1785.6$ : growth rates (in units of $\mathrm{U} / \mathrm{D}$ ) against $R e$. Each line either indicates the locus of a real eigenvalue (solid) or a complex conjugate pair (dashed) as Re changes. The blue (dark) lines correspond to those which are shift-\&-reflect symmetric $\frac{13}{}$ while the green (light) lines are anti-shift-\&-reflect symmetric. The TW itself bifurcates off the mirror-symmetric TW branch near $R e=1770$.

\section{B. Continuation of the RPO}

Continuation of these orbits along the Re-axis is used to produce a bifurcation diagram. Once one RPO is known at a given value of $\delta$, it is straightforward to use it as an initial condition for the Newton-Krylov algorithm at slightly different values of $\delta$. Progression towards larger $\delta$ (i.e. lower $R e$ ) by using small enough steps enables one in principle to track the solution down until the branch possibly bends back.

Trajectories corresponding to RPO solutions have been traced on a two-dimensional plane $\left(D^{\prime}, E^{\prime}\right)$ for various values of $\delta$. Here $D^{\prime}:=R e^{-1} \int\left|\nabla \times \boldsymbol{u}^{\prime}\right|^{2} d^{3} x$ and $E^{\prime}:=\frac{1}{2} \int\left|\boldsymbol{u}^{\prime}\right|^{2} d^{3} x$ are 


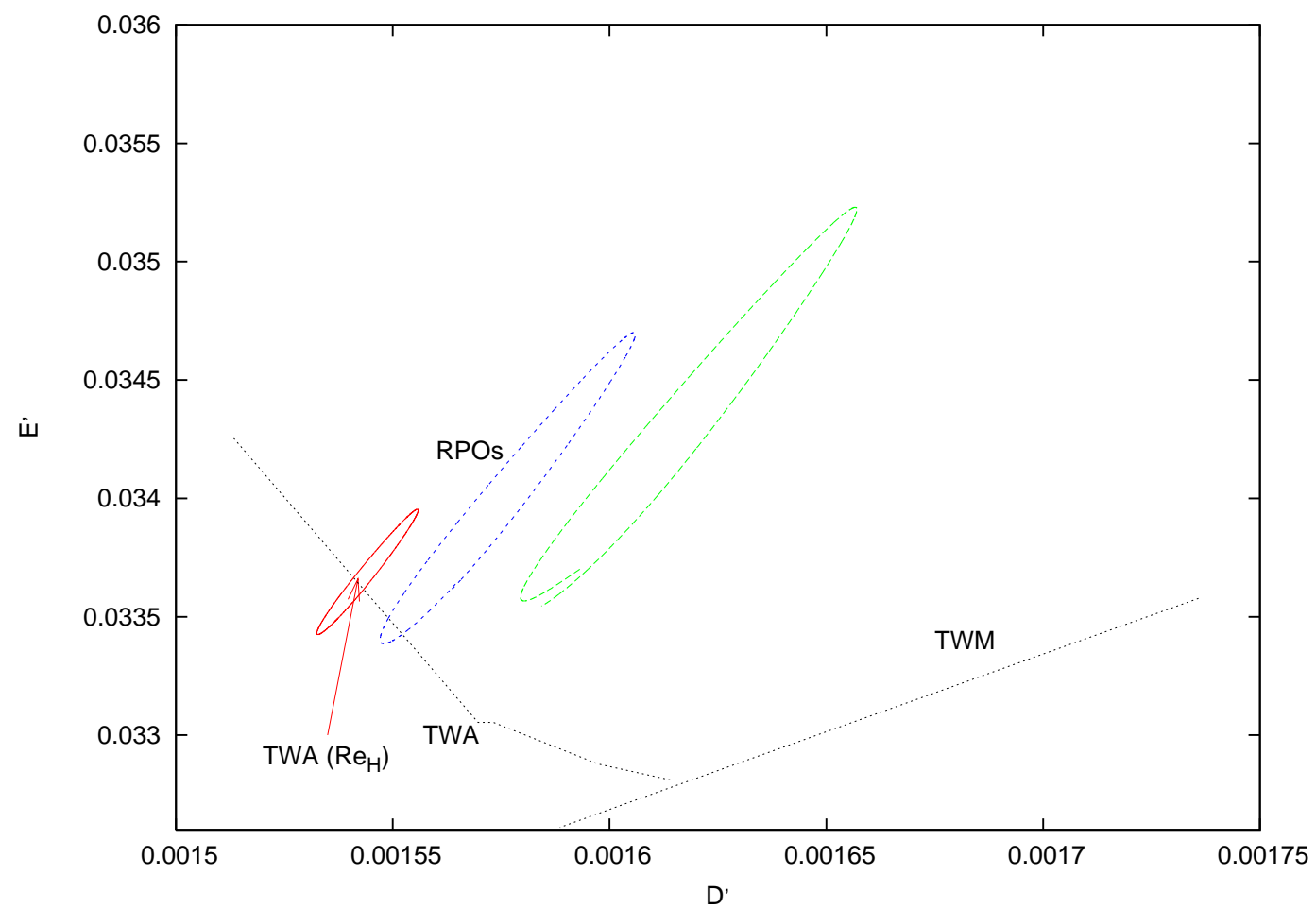

FIG. 5: Numerically found RPOs in the $\left(D^{\prime}, E^{\prime}\right)$ plane for different values of $\delta=4$ (solid red) $\delta=22$ (dotted blue) $\delta=47$ (dashed green), with $\delta=R e_{H}-R e$. The asymmetric (TWA) and mirror-symmetric (TWM) TW branches are also indicated (black dotted curves), parametrised by $R e$. The TWA at $R e=R e_{H}$ is indicated by the red arrow. The RPO corresponding to $\delta=47$ does not look perfectly closed, because of numerical issues detailed in Subsection IIIC. Note that the TWA branch exists only for $R e \geq 1770(\delta \leq 15)$.

respectively the dissipation and the kinetic energy of the disturbance $\boldsymbol{u}^{\prime}:=\boldsymbol{u}-\boldsymbol{u}_{H P}$ to the laminar flow (see Figure 5). Such a projection makes these RPOs look periodic, whereas in the non-moving frame they are only relative periodic orbits. The orbits are slightly elliptic and correspond to a slow modulation of the TW from which they have bifurcated, whereas a TW at a given $R e$ would appear as a dot.

We define their normalized amplitude by :

$$
\delta E=\frac{E_{\max }-E_{\min }}{E_{\max }+E_{\min }}
$$

where the subscripts min and max denote extrema along a cycle of the RPO $(\delta E=0$ for a TW solution). The further $\delta$ is away from the bifurcation point, the larger the amplitude of the RPO, as is expected from a regular Hopf bifurcation. The values of $T$ and $\Delta z$ both 


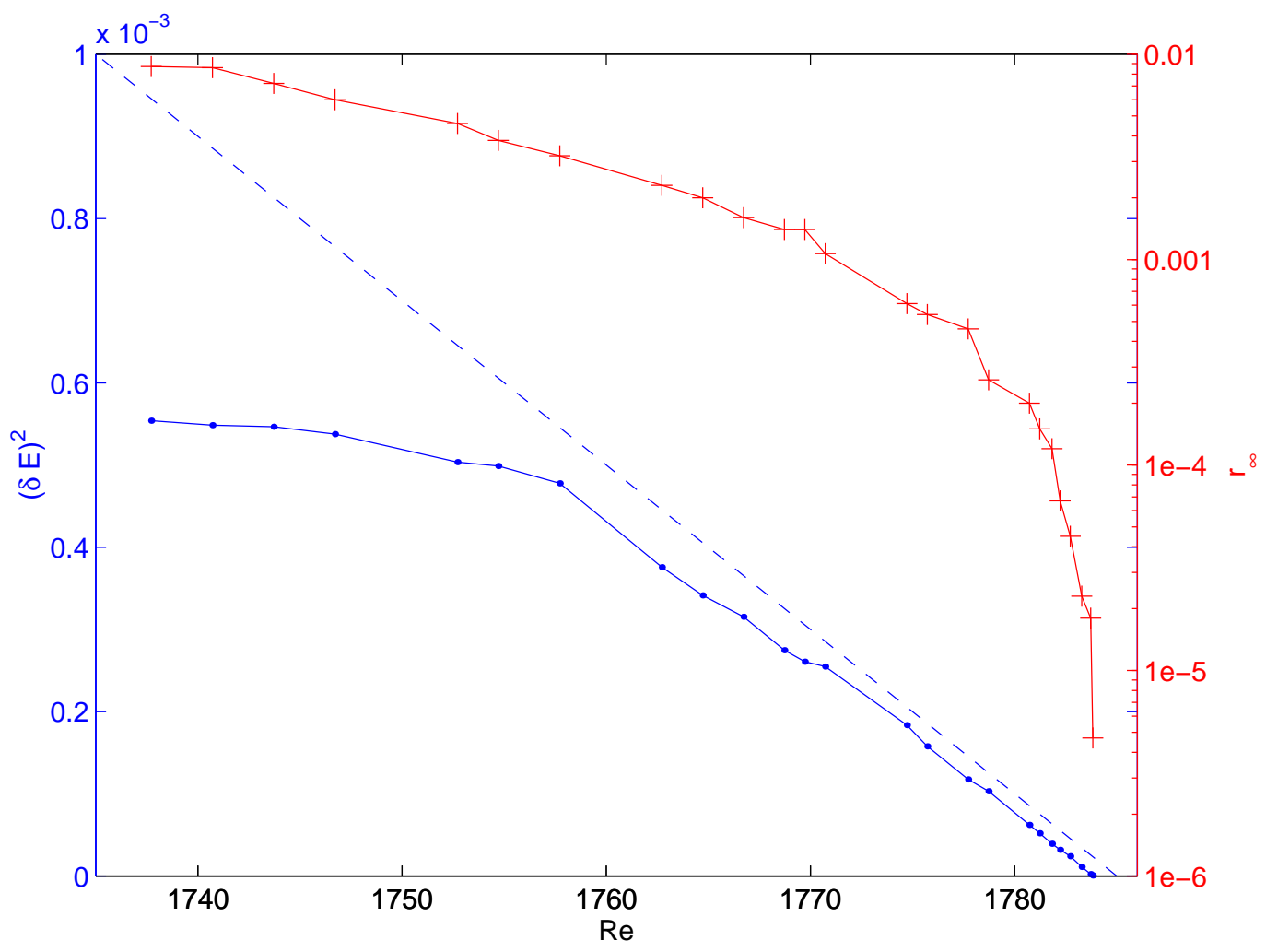

FIG. 6: Normalised amplitude $(\delta E)^{2}$ for the RPO as a function of Re (lower blue data), along with asymptote $(\delta E)^{2}=2 \times 10^{-5} \delta$ where $\delta=R e_{H}-R e$ (blue dashed line). The minimal normalised residual $r_{\infty}=\left|\boldsymbol{X}_{-\Delta z}(T)-\boldsymbol{X}(0)\right| /|\boldsymbol{X}(0)|$ achieved by the Newton-Krylov algorithm is also shown as a function of Re (upper red data). Continuation was stopped when the numerical residual reached $10^{-2}$.

keep the same order of magnitude. For $\delta$ up to 50, the tendency for the orbits is to achieve excursions towards regions of higher dissipation and energy. The energy variations along one cycle stay small for the range of Re considered. Figure 6] shows the dependence of the orbit amplitude $\delta E$ on $R e$ : $\delta E$ grows monotonically with the reduced parameter $\delta$ and is well approximated by the expression $\delta E=O\left(\delta^{\frac{1}{2}}\right)$ near the bifurcation point. The curve has been continued numerically until $\delta=47$. Above this value, convergence becomes questionable see Figure [6 - as discussed in Subsection [IIC and in the conclusion.

The value of $\Delta z$ indicates the distance travelled by the exact coherent structure as it propagates down the pipe in time $T$. This defines a phase speed for the RPO,

$$
c_{R P O}:=\Delta z / T
$$




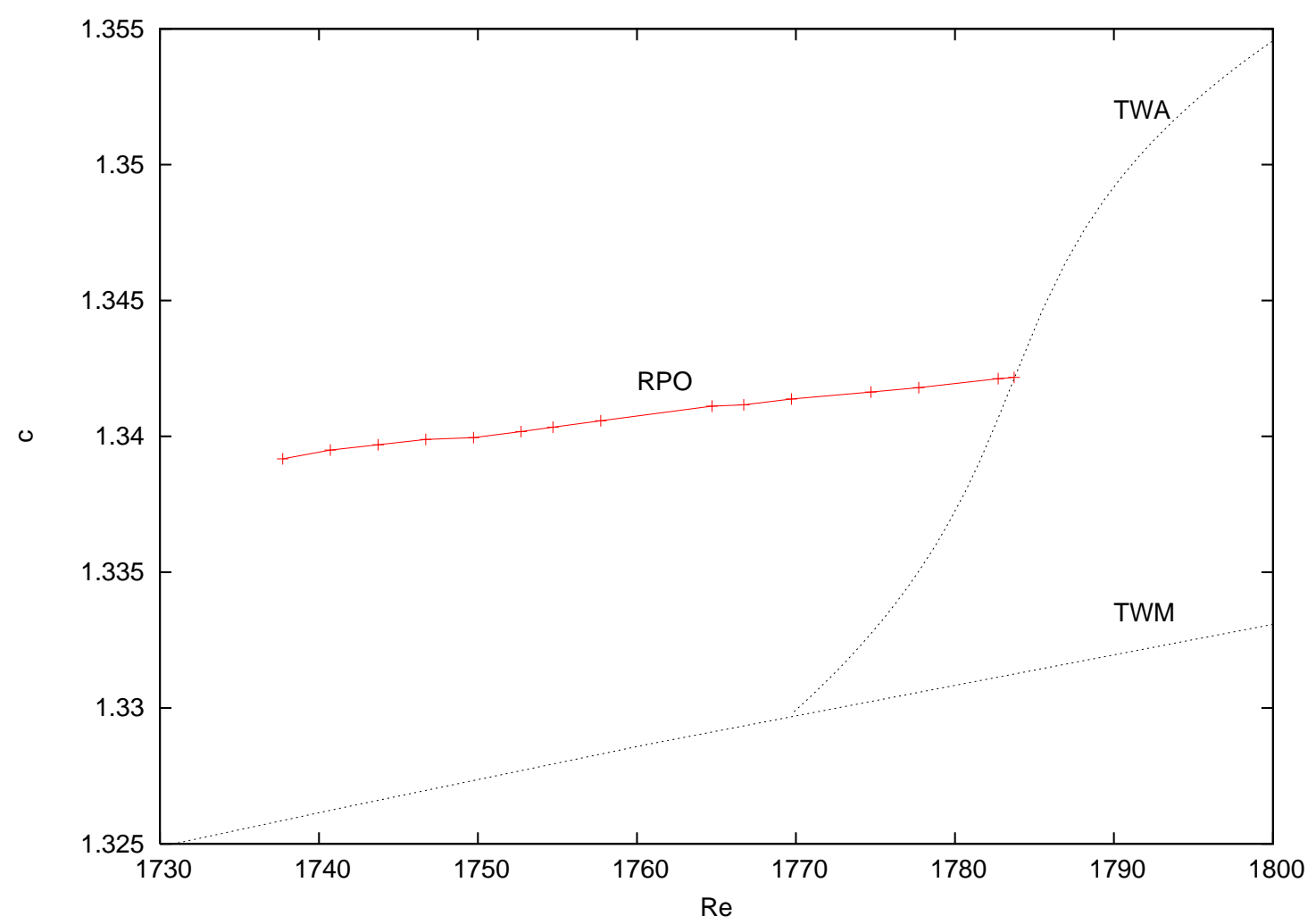

FIG. 7: Axial phase velocity $c$ (in units of $U$ ) vs. Re for the RPO (red), the asymmetric TW (TWA) and the mirror-symmetric TW (TWM) (both in black).

corresponding to the axial speed of the frame in which the orbit would be exactly periodic. Figure 7 is a diagram showing the phase velocity of both the asymmetric TW branch and the mirror-symmetric TW branch, compared to the phase velocity of the RPO which varies little over the range of Re considered. Note that the RPO exists at values of Re below that at which the asymmetric TW appears (see Figure 7).

Figure 8 shows the evolution of the velocity field in a cross-section moving with the speed $c_{R P O}$ at $\delta=47$ (i.e. $R e \sim 1738$ ). This moving frame is chosen to emphasize slow-time variations. Near the left of the cross-section, the flow is strongly reminiscent of the pattern of the original asymmetric TW, which would give 4 identical slices. There is a slight periodic modulation of the shape and position of the low-speed streak. The high-speed streaks close to the wall look more robust in shape during a cycle, but their intensity fluctuates. As $\delta$ increases, the intensity inside the patches of axial velocity on the right of Figure 8 fluctuates more in time than the ones of the left. The larger $\delta$, the more symmetric the cross-sectional pattern looks (this feature is also shared with the asymmetric TW branch as it approaches 

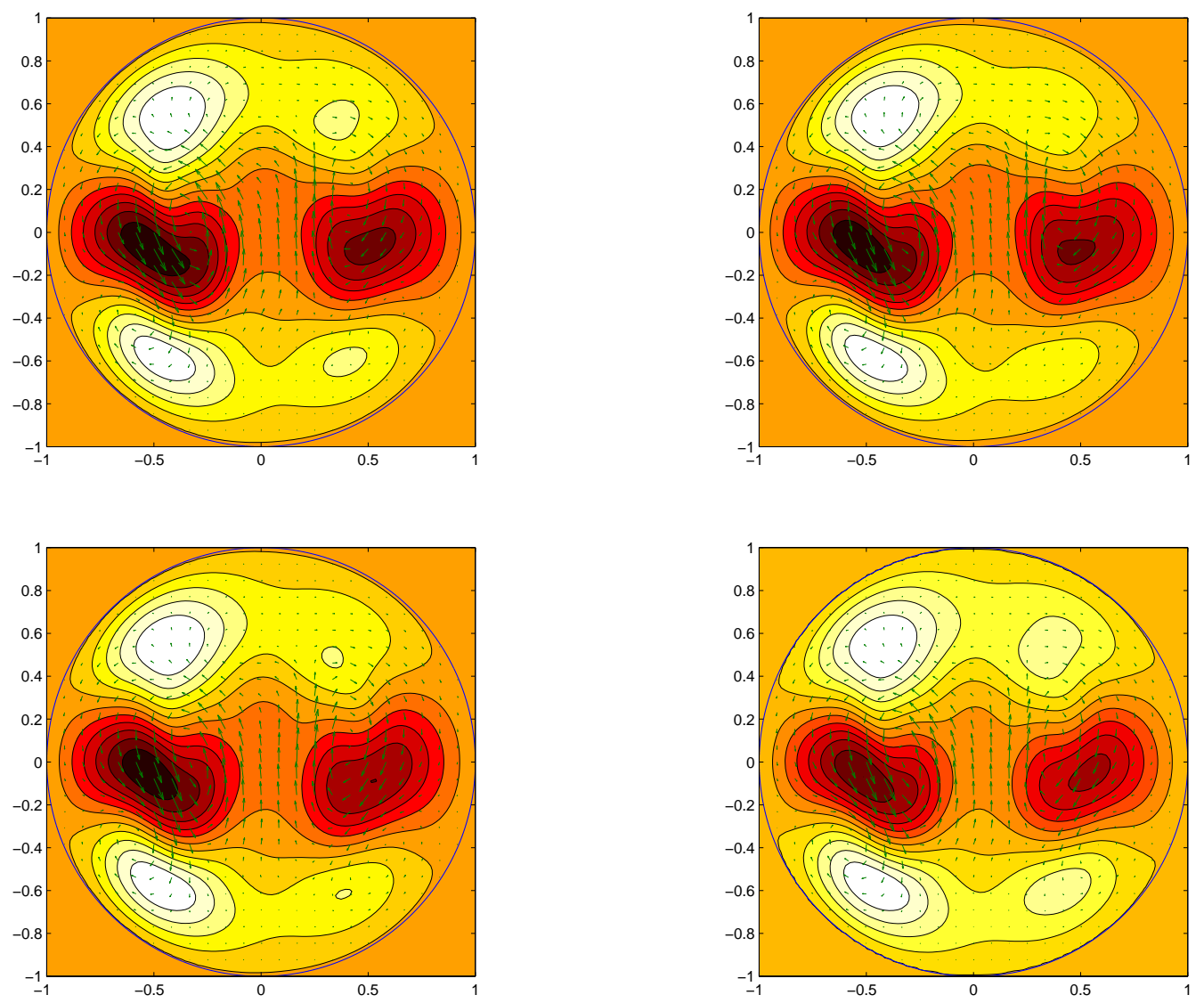

FIG. 8: Velocity field of the RPO in the moving frame, at a cross-section defined by $z-c_{R P O} t=0$ (see Eq. 21), for $\delta=47$ (Re=1738). From left to right, from top to bottom : $t=7.2, t=18.7$, $t=30.3$ and $t=41.8$ in units of $d / U$. The colours and arrows are as in Figure 3 .

the mirror-symmetric branch). The cross-section velocity is more steady than the axial velocity, except in the vicinity of the weaker low-speed streak on the right of the pipe, where the vortex looks 'attached' to the streak.

Figure 9 displays the friction factor $\Lambda$, defined using dimensional variables as

$$
\Lambda:=-\frac{d p}{d z} /\left(\frac{1}{2} \rho U^{2}\right)
$$

of the RPO branch. For any given value of $R e, \Lambda$ along a cycle is always below those of the asymmetric and mirror-symmetric TW solutions. The highest value $\Lambda_{\max }$ reached during a cycle gets closer to that of the mirror-symmetric branch as Re is decreased. 


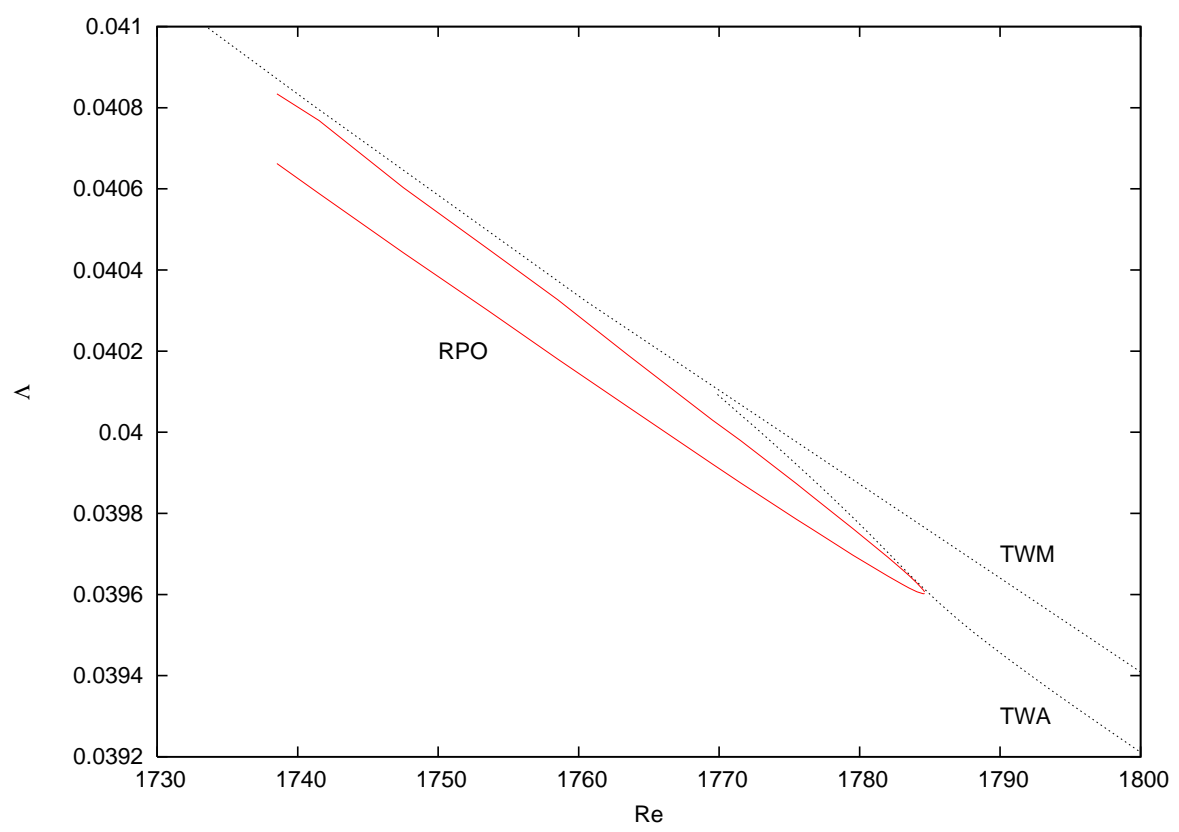

FIG. 9: Friction factor $\Lambda$ vs. Re for the RPO (red), the asymmetric TW and the mirror-symmetric TW (both black). In the case of the RPO, only the extremal values $\Lambda_{\min }$ and $\Lambda_{\max }$ over a cycle are shown.

\section{Instability of the RPO and numerical issues}

While performing the continuation in $\delta$, we observed that $r_{\infty}$ (the value around which the numerical residual stagnates) increases steadily with $\delta$. This trend is clear from Figure 6. While animation of the velocity field does not show any discontinuity in time for $\delta>25$, the projection on the $\left(D^{\prime}, E^{\prime}\right)$ plane no longer looks perfectly closed (see Figure 5 ). We deliberately stopped the continuation near $\delta \sim 50$ when it was difficult to reduce $r_{\infty}$ below $10^{-2}$.

In order to understand this behaviour of $r_{\infty}$, we first need an idea of how accurate the search for RPOs is expected to be. The minimum residual $r_{\infty}$ is limited by both the accuracy of the time-stepping scheme and the natural instability of the trajectory. Any component of any point on the numerically found RPO is accurate to at least $O\left(\epsilon_{m}\right)$, the machine precision. After a period $T$, this numerical discrepancy has increased by a factor $e^{\lambda T}$, with $\lambda$ being now the largest Floquet exponent of the RPO. Indeed a useful rule-of-thumb for 
estimating the lowest residual attainable is:

$$
r_{\infty} \sim O\left(n \epsilon_{m} e^{\lambda T}\right)
$$

We can illustrate this formula by simply time-stepping from a sufficiently converged asymmetric TW at $\delta=0$. After the TW has travelled one single pipe length, $r(t=L / c) \sim 10^{-10}$; whereas after a time $T$ (the period of the RPO), $r(T) \sim 10^{-5}$. Using expression (23), we can estimate $\lambda_{T W} \sim \ln \left(10^{5}\right) / T \sim 0.26 U / d$. Sufficiently close to the Hopf bifurcation, both the RPO and the TW are expected to have the same leading Floquet exponent $\lambda$, hence the estimation $\lambda_{R P O}(\delta=0) \sim 0.26 U / d$. Since the period $T$ does not vary much (less than $1 \%$ ) within the range of $\delta$ considered, we can extract from the data in Figure 6 a trend for the Floquet exponent of the RPO. We found the following scaling : $\lambda_{R P O}(\delta) \sim O\left(\delta^{\gamma}\right)$, with $\gamma$ very close to $\frac{1}{8}$. Unsurprisingly, the RPO is more unstable away from the bifurcation point. A possible explanation is the stronger slow-time modulation of the streaks (whose strong transversal velocity gradients are already responsible for the inertial instability of the TW) as $\delta$ increases. This harmonic modulation of the streaky field over a full cycle is likely to increase the instability of the whole flow.

\section{CONNECTION WITH A TW SOLUTION}

A leading question is how the RPO found in this paper is related to transition to turbulence in a pipe. The asymmetric TW is already known to play a special role. Firstly, it lies on the laminar-turbulent boundary (also called the 'edge of chaos ${ }^{37}, 38$ ), which is the set of initial conditions separating trajectories which directly relaminarise from those which lead to turbulent behaviour. This means that infinitesimal perturbations to the TW can either lead to transition or relaminarisation, depending on their exact form. Secondly, recent studies have shown that for analogous parameters, but larger $R e$, this asymmetric TW is generally the only exact travelling wave solution closely visited by trajectories when constrained on the laminar-turbulent boundary. ${ }^{23}$ It is thus interesting to see if other kind of exact recurrent states, like the RPO found here fulfil the expectation of: (a) lying on the laminar-turbulent boundary as well; (b) connecting to other states via heteroclinic connections; and (c) getting transiently approached by transitional trajectories. 
We have chosen to concentrate on $\delta=2(R e=1783.6)$, and perturbed the RPO by two random vectors in the following manner :

$$
\boldsymbol{X}(t=0)=\boldsymbol{X}_{R P O}+\epsilon\left(\cos \phi \boldsymbol{e}_{1}+\sin \phi \boldsymbol{e}_{2}\right) .
$$

In this expression, $\epsilon$ is a small parameter (here $10^{-4}$ ), $\boldsymbol{e}_{1}$ and $\boldsymbol{e}_{2}$ are two random vectors of norm unity, which are generic enough to possess non-zero components along the unstable manifold of the RPO. $\phi$ is a shooting angle which has been varied in the range $[0,2 \pi]$. We notice that several trajectories starting from the new state $\boldsymbol{X}(t=0)$ relaminarise directly while other trajectories undergo a short 'turbulent' transient, indicated by a dramatic rise in the kinetic energy and dissipation of the disturbance to the laminar flow, and by a loss of symmetry of the flow. Hence the RPO found for $R e=1783.6$ is also on the laminar-turbulent boundary.

Establishing heteroclinic connections between the RPO and other exact coherent structures is an involved and technically demanding task. We can, however, seek suggestive evidence for such links by exploring the evolution of trajectories originating close to the RPO and confined to remain in the laminar-turbulent boundary. This was attempted for the $\mathrm{RPO}$ at $\delta=2$ by refining the angle $\phi$ defined in (24) to find a phase-space trajectory which stays near the laminar-turbulent boundary, i.e. neither directly relaminarises nor undergoes a turbulent transient. This is done via shooting method based on bisection of the value of $\phi$. Refining $\phi$ up to three significant digits results in a trajectory called $H_{1}$, which eventually relaminarises after a significantly long transient along the laminar-turbulent boundary. For a short duration during the transient (less than $10 \mathrm{~d} / U$ ), all velocity components oscillate with the same apparent period on a short-time scale, while the energy reaches a plateau. Based upon previous experience $e^{\underline{23}}$, we recognised this as the signature of a travelling wave solution lying nearby in phase space. The scalar function

$$
r_{\min }(t)=\min _{t^{\prime}>t}\left\{r_{t}\left(t^{\prime}\right), \mid \frac{\partial r_{t}}{\partial t^{\prime}}=0\right\}
$$

where

$$
r_{t}\left(t^{\prime}>t\right)=\frac{\left|\boldsymbol{X}\left(t^{\prime}\right)-\boldsymbol{X}(t)\right|}{|\boldsymbol{X}(t)|} .
$$

was calculated along the whole trajectory. This function, closely linked to $|\boldsymbol{G}|$, measures how recurrent a flow is at a given location in space (ignoring the possibility of shifted 
recurrences). Figure 10 shows $r_{\text {min }}$ as a function of the starting point on the edge trajectory $H_{1}$. While $t \leq 60 d / U, r_{\min }$ is very low $\left(\mathrm{O}\left(10^{-3}\right)\right)$ and the flow is nearly recurrent. The slow-time modulation reflects the modulation of the flow along the RPO. Later the trajectory leaves the neighbourhood of the RPO because of its instability, and $r_{m i n}$ increases up to large values of $\approx 0.2$. At a later phase corresponding to the plateau in energy, $r_{\min }(t)$ displays a clear dip (labelled N1 in Figure 10) down to values of $\approx 5 \times 10^{-2}$, and then increases again. Such low values of $r_{\min }$ are never reached if the angle $\phi$ is chosen randomly and indicate that the edge trajectory has entered the (phase-space) vicinity of an exact periodic solution, at which $r_{\min }$ vanishes. The starting point at $t \sim 150$ which yielded the lowest $r_{\min }$ was used as an initial state for the Newton-Krylov algorithm with $\Delta z=2 \pi / \alpha$ and $\Delta \theta=0$. This readily converged to the asymmetric TW, shifted azimuthally by a half turn, with $r_{\min } \sim 10^{-10}$.

Shooting in the opposite direction, then refining properly the angle $\phi$, leads to another 'edge' trajectory $H_{2}$. The corresponding recurrence function $r_{\min }(t)$ is plotted in Figure 10. The features of $r_{\min }$ are reminiscent of those of the trajectory $H_{1}$. A dip (labelled N2) appears near $t \approx 190 \mathrm{~d} / U$, and the corresponding state has been used as a starting point for the Newton-Krylov algorithm (again we look explicitly for a TW solution). The algorithm converged to another TW solution, that we call 2b_1.5. Close examination revealed that it is equivalent of the TW 2b_1.25 mentioned in Kerswell \& Tutty $\underline{\underline{16}}$ and Duguet et. al. $\stackrel{23}{\text {, }}$ but with an axial wavenumber of $\alpha=1.5$ instead of $\alpha=1.25$. Here $\alpha=0.75=1.5 / 2$, so that exactly two wavelengths of the TW fill the whole domain. This TW solution was already mentioned in Duguet et. al. $\frac{23}{3}$ because it is an attractor for edge trajectories when $R e=2400, \alpha=1.25$, and when the flow is constrained to a 2-fold rotationally symmetric subspace. This attracting property is lost when no rotational symmetry is forced, which explains why the TW here first attracts and then repels the edge trajectory $H_{2}$.

Given the difficulty of getting the Newton-Krylov algorithm to converge in high dimensions unless the starting guess is sufficiently close to a solution, it is reasonable to assume that the above trajectories enter the neighbourhood of the TWs before ultimately relaminarising. Since the unstable manifold of these TWs in the laminar-turbulent boundary is of such small dimension, it is tempting to speculate that these visits are actually indicative 


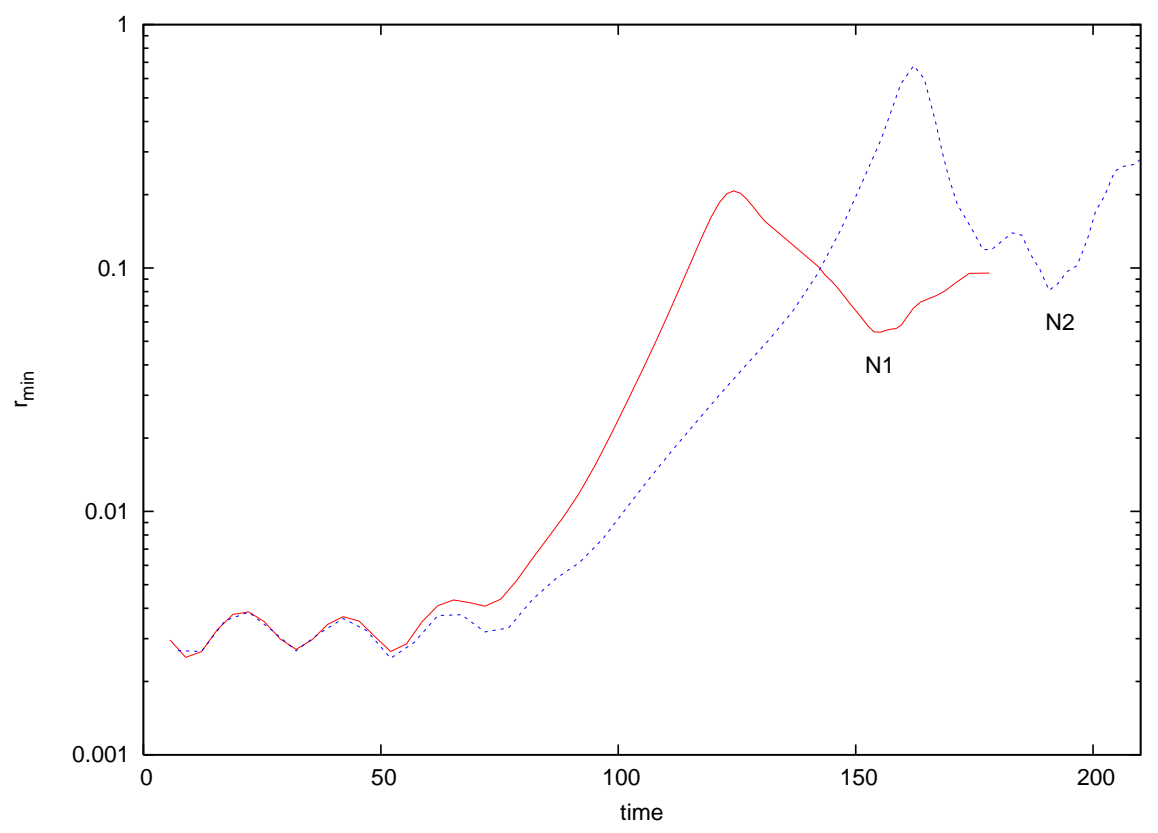

FIG. 10: Recurrence function $r_{\min }$ vs. time for the two 'edge' trajectories $H_{1}$ (red solid) and $H_{2}$ (blue dotted), starting from the perturbed RPO and constrained to the laminar-turbulent boundary. The dip labelled N1 (resp.N2) near $t \sim 150$ (resp. $t \sim 190$ ) indicates an approach towards a TW state.

of heteroclinic connections linking the RPO to the two TWs. Establishing this formally is a significant undertaking not pursued further here but the idea that trajectories link different coherent structures to produce a saddle structure on the laminar-turbulent boundary is consistent with other recent observations. $\underline{23}$

The trajectories $H_{1}$ and $H_{2}$ are projected on a two-dimensional $(I, D)$ diagram in Figure 11. $I$ and $D$ are respectively the energy input and the total viscous dissipation in the computational domain, defined by $I:=\oint p \boldsymbol{u} \cdot \boldsymbol{n} d S, D:=R e^{-1} \int|\boldsymbol{\nabla} \times \boldsymbol{u}|^{2} d^{3} x$. These two quantities are related by the energy equation

$$
\frac{d E}{d t}=I-D
$$

where $E$ is the total kinetic energy $E:=\frac{1}{2} \int|\boldsymbol{u}|^{2} d^{3} x$. Hence (relative) equilibria are all located on the diagonal $I=D$. $I$ and $D$ are normalised in Figure 11 by $\bar{I}$, which is the value of $I$ for the laminar flow (hence located here at $(I, D)=(1,1)$ ). Both trajectories $H_{1}$ and $H_{2}$ escape from the RPO solution along its unstable manifold, in a direction locally tangent to the laminar-turbulent boundary. They reach much larger values of $D$ and $I$, before turning 


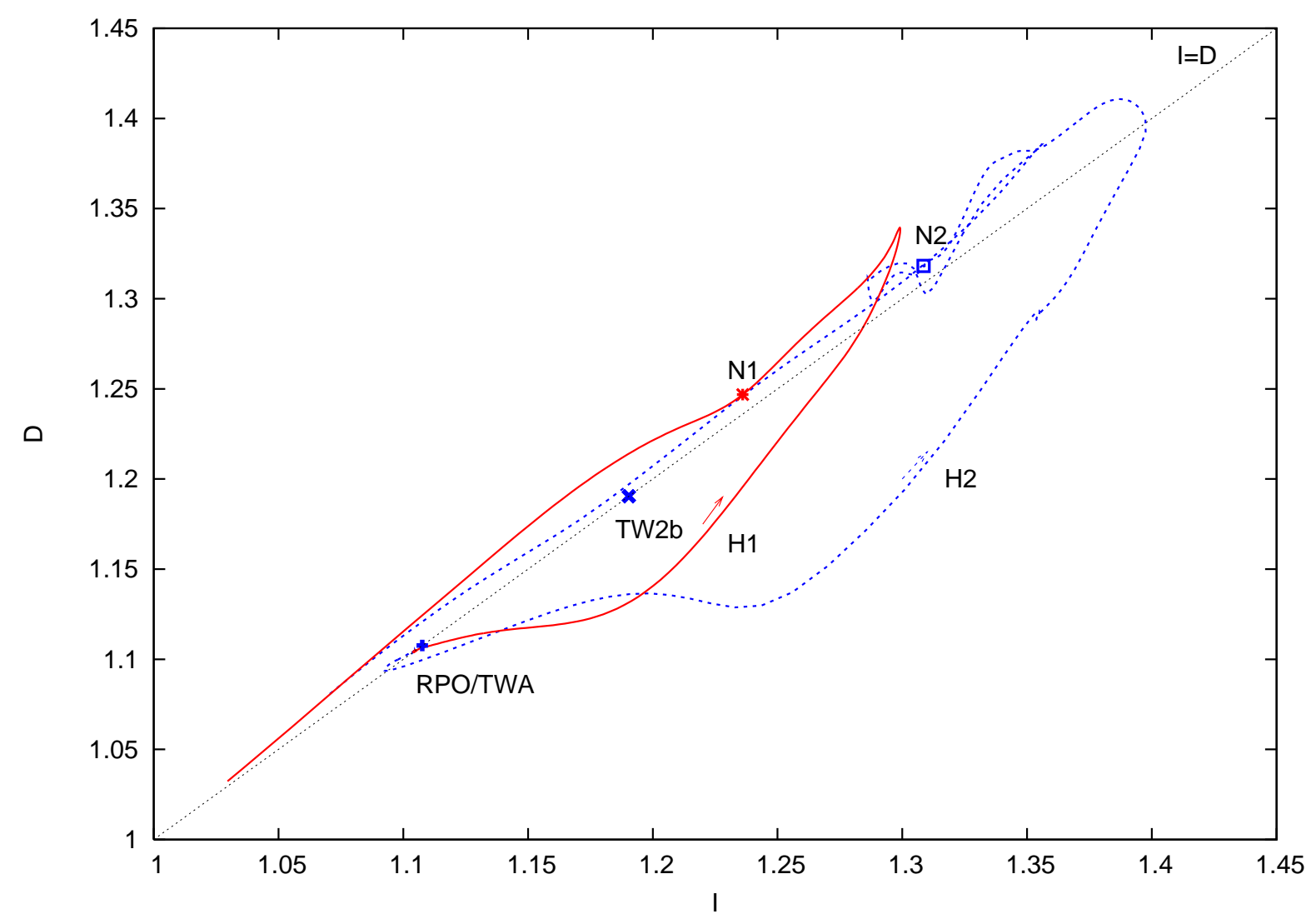

FIG. 11: Normalised $(I-D)$ projection of the 'edge' trajectories $H_{1}$ (red) and $H_{2}$ (blue) starting from the perturbed RPO, $R e=1783.6$. Also shown are the two TW states (blue cross and dot) identified using the Newton-Krylov algorithm : TWA stands for the Asymmetric TW, TW2b for the TW solution 2b_1.5. The location of the RPO is undistinguishable from that of the Asymmetric TW on this plot.

back towards the laminar state, each of them passing by the vicinity of one of the TWs. The time of closest approach in the $(I, D)$ plane does not coincide with the minima of $r_{\min }$ (e.g. $N 2$ on $H 2$ is not the closest point of approach to $2 b_{-} 1.5$ in the $(I, D)$ plane). This difference, of course, highlights the ongoing dilemma of how to select the 'right' norm to measure distances in the infinite-dimensional phase space associated to the Navier-Stokes equations. 


\section{CONCLUSION}

We have shown that it is possible to numerically capture relative periodic orbits (RPOs) in pipe flow when the associated dynamical system size exceeds $10^{5}$ degrees of freedom. We have applied successfully our method to find a RPO branch in the vicinity of the Hopf bifurcation occurring along the asymmetric Travelling Wave branch for $\alpha=0.75$. This exact coherent structure consists of a streaky pattern which is modulated over one period of roughly $43 \mathrm{~d} / \mathrm{U}$, while travelling approximately 60 pipe diameters downwards in the axial direction.

Further evidence is presented here that the laminar-turbulent boundary is structured around of network of exact solutions linked to each other by heteroclinic and homoclinic connections $1,16,23,34$. For $L \sim 5 d(\alpha=0.625)$ and $R e=2875$, asymmetric TW solutions are recurrently approached by laminar-turbulent trajectories; ${ }^{23}$ here for $L \sim 4.19 d(\alpha=0.75)$ and $R e=1783.6$, the same conclusion seems to hold. However, now this schematic view of edge trajectories recurrently visiting neighbourhoods of TWs needs to be extended to take into account relative periodic orbits. All these solutions and their stable manifolds make up the laminar-turbulent boundary.

The main problem in accurately identifying RPOs is the exponential divergence of a nearby orbit from the RPO. In practice, the instability of the RPO together with its typically long period lead to a large value for its leading characteristic multiplier. This causes a significant loss of numerical accuracy when searching for neighbouring orbits which almost close on themselves. This effect was found to worsen away from the bifurcation point as the RPO becomes more unstable so that it was only possible to trace the RPO branch accurately in a relatively small neighbourhood of the bifurcation point. As a result, its amplitude remains small and the RPO dynamics is close to that of the underlying TW solution. The original goal of tracing the RPO down (in $R e$ ) to a likely saddle node bifurcation point and then exploring the 'other' branch have, unfortunately, been out of reach. Whether this 'other' branch reconnects to another TW branch or becomes a more nonlinear unstable object possibly embedded in the flow's turbulent dynamics remains a challenge for the future. There are ways to improve the numerical algorithm such as a multiple-shooting technique 
where the orbit is subdivided into manageable pieces which now clearly need to be explored.

Physically, the instability of the RPO means that phase-space trajectories are unlikely to spend a long time in its vicinity, despite the likelihood that the RPO is linked via heteroclinic connections to other more attracting solutions. In particular, the probability is low that such a solution could be identified experimentally, despite recent progress made in flow visualisation or indeed numerically as part of a direct numerical simulation. The same conclusion may well apply to all RPOs of the system.

At the start of this study, the stability of various lower TW branches was considered in order to identify how frequently Hopf bifurcations of TW branches arise. For $\alpha=0.75$, the asymmetric TW branch undergoes only one Hopf bifurcation below $R e=5000$ which is examined in this paper. The mirror-symmetric branch displays another one near $R e=3487$, its period near the bifurcating point is $T=68$, which makes it even more unstable and harder to continue than the previous one. For the same parameters, we have checked that another known branch of TW (referred to as 2b_1.25 $\frac{16.23}{}$ ) does not display any Hopf bifurcations at all below $R e=3400$. Despite the fact that many more TWs might exist, it appears that Hopf bifurcations of lower-branch TW solutions are not frequent, and that short-period RPOs associated with them are not generic objects. From this point of view, the fact that mainly TW solutions have been identified during transition does not mean that RPOs do not participate in the transitional dynamics, but rather that their contribution is likely to be more infrequent and fleeting. However, it might not be the case for other shear flows such as plane Couette flow where (non-relative) periodic orbits also bifurcate from a lower-branch steady state. $\stackrel{34,39,40}{ }$ This does not exclude either the existence of RPO solutions entirely disconnected from the TW solutions, which would appear via saddle-node bifurcations. Such a (periodic) solution has been computed in plane Couette flow, and its dynamics is embedded in the turbulent dynamics $\underline{20} \underline{21}$ The numerical method described in Section III is an adequate tool to seek them providing a good enough starting point is identified for the Newton-Krylov algorithm. 


\section{Acknowledgments}

We would like to thank A.P. Willis for sharing his time-stepping code with us and for stimulating comments during this work. Y.D. is supported by a Marie-Curie Intra European Fellowship (grant number MEIF-CT-2006-024627). C.C.T.P. is supported by an EPSRC grant.

1 O. Reynolds. An experimental investigation of the circumstances which determine whether the motion of water shall be direct or sinuous and of the law of resistance in parallel channels. Phil. Trans. Roy. Society, 174:935-982,1883

2 A. Darbyshire, T. Mullin. Transition to turbulence in constant mass-flux pipe flow. J. Fluid Mech. , 289:89-114,1995

3 W. Pfenniger. Transition in the inlet length of tubes at high Reynolds numbers Boundary Layer and Flow Control (ed.GV Lachman), 970, 1961

4 P.G. Drazin, W.H. Reid. Hydrodynamic Stability Cambridge University Press,1985

5 F. Waleffe. Three-dimensional coherent states in plane shear flows. Phys. Rev. Letters, 81(19):4140-4143, 1998

6 T. Mullin, R.R. Kerswell (eds). Non-Uniqueness of solutions ot the Navier-Stokes equation and their connection with laminar-turbulent transition. Springer-Verlag,2004

7 R.R. Kerswell. Recent progress in understanding the transition to turbulence in a pipe. Nonlinearity, 18:17-44, 2005

8 J.F. Gibson, J. Halcrow, P. Cvitanović The geometry of state space in plane Couette flow accepted for publication in J. Fluid Mech., 2008

9 J. Kim, J. Hamilton, F. Waleffe. Regeneration mechanisms of near-wall turbulence structures. J. Fluid Mech., 287:317-348, 1995

10 F. Waleffe Exact Coherent Structures in Channel Flow, J. Fluid Mech., 435:93-102, 2001

11 M. Nagata. Three-dimensional finite-amplitude solutions in plane Couette flow : bifurcation from infinity. J. Fluid Mech., 217:519-527, 1990

12 H. Faisst, B. Eckhardt. Traveling waves in pipe flow. Physical Review Letters, 91:224502, 2003

13 H. Wedin, R.R. Kerswell. Exact coherent structures in pipe flow : travelling wave solutions. $J$. 
Fluid Mech., 508:333-371, 2004

14 B. Hof, C.W.H. van Doorne, J. Westerweel, F.T.M. Nieuwstadt, H. Faisst, B. Eckhardt, H, Wedin, R.R. Kerswell, F. Waleffe. An experimental observation of travelling waves in pipe flow Science, 305, 1594-1597, 2004

15 B.Hof, C.W.H. vanDoorne, J.Westerweel, F.T.M. Nieuwstadt. Turbulence regeneration in pipe flow at moderate Reynolds numbers. Phys. Rev. Lett., 95, 214502,2005

16 R.R. Kerswell, O.R. Tutty. Recurrence of travelling waves in transitional pipe flow. J. Fluid Mech., 584:69-102, 2007

17 T.M. Schneider, B. Eckhardt, J. Vollmer Statistical analysis of coherent structures in transitional pipe flow Phys. Rev. E , 75, 066313, 2007

18 F. Christiansen, P. Cvitanović, V. Putkaradze. Spatiotemporal chaos in terms of unstable recurrent patterns Nonlinearity, 10:55-70, 1997

19 V. Lopez, P. Boyland, M.T. Heath, R.D. Moser. Relative Periodic Solutions of the Complex Ginzburg-Landau Equation SIAM J. Applied Dynamical Systems, 4;1042-1075, 2005

20 G. Kawahara, S. Kida. Periodic motion embedded in plane Couette turbulence: regeneration cycle and burst. J. Fluid Mech., 449:291-300, 2001

21 D. Viswanath. Recurrent motions wihtin plane Couette turbulence. J. Fluid Mech., 580:339-358, 2007

22 C.C.T. Pringle, R.R. Kerswell. Asymmetric, helical and mirror-symmetric travelling waves in pipe flow. Phys. Rev. Letters, 99:074502, 2007

23 Y. Duguet, A.P. Willis, R.R. Kerswell. Transition in pipe flow : the saddle structure on the boundary of turbulence. accepted for publication in J. Fluid Mech., 2008

24 J. Peixinho, T. Mullin Decay of turbulence in pipe flow Phys. Rev. Lett. 96, 094501 (2006)

25 J. Peixinho, T. Mullin Finite-amplitude thresholds for transition in pipe flow. J. Fluid Mech., 582:169-178, 2007

26 A.P. Willis, R.R. Kerswell. Critical behaviour in the relaminarisation of localised turbulence in pipe flow. Phys. Rev. Lett., 98:014501, 2007

27 A.P. Willis, R.R. Kerswell. Coherent structures in localised and global pipe turbulence. Phys. Rev. Lett., 100:0124501, 2008

28 A.P. Willis, R.R. Kerswell. Turbulent dynamics of pipe flow captured in a reduced model: puff relaminarisation and localised 'edge' states. resubmitted (decision likely by proofs), 2008 
29 F. Marquès. On boundary conditions for velocity potentials in confined flows : Application to Couette flow. Phys. of Fluids A, 2(5):729-737, 1990

30 Y. Saad, M. Schultz. GMRES: A generalized minimal residual algorithm for solving nonsymmetric linear systems. SIAM J. Sci. Stat. Comput., 7:1-14, 1986

31 C. Eisenstat, H. Walker. Choosing the forcing terms in an inexact Newton method. SIAM J. Sci. Comput., 17:16-32, 1996

32 H. Walker, R.S. Tuminaro, J.N. Shadid. On backtracking failure in Newton-GMRES methods with a demonstration for the Navier-Stokes equations. J. Comp. Physics, 180:549-558, 2002

33 J.E. Dennis, R.B. Schnabel. Numerical Methods for Unconstrained Optimization and Nonlinear Equations. SIAM Classics, 1996

34 J. Wang, J. Gibson, F. Waleffe Lower branch coherent states in shear flows: Transition and control. Phys. Rev. Lett. , 98, 204501, 2007

35 R. Lehoucq, K. Maschhoff, D. Sorensen, C. Yang. ARPACK Homepage http://www.caam.rice.edu/software/ARPACK/

36 Y. Kuznetsov. Elements of Applied Bifurcation theory. Springer-Verlag, 2004

37 B. Eckhardt, T.M. Schneider, B. Hof, J. Westerweel Turbulence Transition in Pipe Flow. Ann. Rev. of Fluid Mech., 39: 447-468, 2007

38 T.M. Schneider, B. Eckhardt, J.A. Yorke Turbulence Transition and the edge of chaos in Pipe Flow. Phys. Rev. Lett. , 99, 034502, 2007

39 G. Kawahara. Laminarization of minimal plane Couette flow : Going beyond the basin of attraction of turbulence Phys. Fluids, 17, 041702, 2005

40 G. Kawahara, S. Kida, M. Nagata. Unstable Periodic Motion in plane Couette System: The Skeleton of Turbulence. IUTAM Symposium on One Hundred Years of Boundary Layer Research, 129:415-424, 2006 